\title{
A guide to immunotherapy for COVID-19
}

\author{
Frank L. van de Veerdonk $\oplus^{1 凶}$, Evangelos Giamarellos-Bourboulis ${ }^{2}{ }^{2}$, Peter Pickkers ${ }^{3}$, \\ Lennie Derde ${ }^{4}{ }^{4,9}$, Helen Leavis ${ }^{5}$, Reinout van Crevel', Job J. Engel', W. Joost Wiersinga ${ }^{6}$, \\ Alexander P. J. Vlaar ${ }^{7}$, Manu Shankar-Hari $\oplus^{8}$, Tom van der Poll ${ }^{6}{ }^{6}$, Marc Bonten ${ }^{9}$, Derek C. Angus ${ }^{10}$, \\ Jos W. M. van der Meer ${ }^{1}$ and Mihai G. Netea ${ }^{1,11 凶}$
}

\begin{abstract}
Immune dysregulation is an important component of the pathophysiology of COVID-19. A large body of literature has reported the effect of immune-based therapies in patients with COVID-19, with some remarkable successes such as the use of steroids or anti-cytokine therapies. However, challenges in clinical decision-making arise from the complexity of the disease phenotypes and patient heterogeneity, as well as the variable quality of evidence from immunotherapy studies. This Review aims to support clinical decision-making by providing an overview of the evidence generated by major clinical trials of host-directed therapy. We discuss patient stratification and propose an algorithm to guide the use of immunotherapy strategies in the clinic. This will not only help guide treatment decisions, but may also help to design future trials that investigate immunotherapy in other severe infections.
\end{abstract}

$\mathrm{T}$ he COVID-19 pandemic forced the world to accelerate vaccine and drug development and evaluation at an unparalleled pace. At present, the COVID-19 treatment armamentarium is largely represented by antiviral agents (often administered in early stages of disease) and immunotherapeutic agents that modulate the host immune response (often administered in more advanced stages of disease) - with the rationale for immunotherapy being that dysregulation of host responses feature prominently in COVID-19 pathophysiology. Host-directed therapy is, however, a relatively complex approach, and several important aspects need to be considered.

First, apparently obvious choices based on knowledge extrapolated from analogous conditions may be inappropriate in the face of novel diseases with complex immunopathology. Indeed, the initial expert opinion to avoid corticosteroids as immunomodulatory treatment for COVID-19, while later on they became standard-of-care (SoC), underscores the importance of obtaining solid evidence based on robust clinical trials. Second, the hostpathogen response and resulting immunological milieu is very heterogenous, which indicates that not every patient will benefit from the same immunomodulatory treatment strategy. Furthermore, this heterogeneity may not be clinically evident at the bedside, potentially necessitating the evaluation and deployment of biomarkers to guide patient-specific immune therapy. Third, all of this complexity needs to be dissected, understood, and then re-packaged in updated treatment algorithms in a setting of constant change in available evidence.

Here, we attempt to provide guidance for immunotherapy of patients with COVID-19, on the basis of consideration of these three major points. We will provide an overview of evidence from the major clinical trials of host-directed therapy, discuss patient stratification, and propose an algorithm to guide the use of immunotherapy strategies.

\section{Immune pathophysiology of COVID-19}

COVID-19 is a complex disease in which respiratory manifestations associated with viral replication are accompanied by systemic effects, indicating that SARS-CoV-2 infection is likely to generate a broadly dysregulated immune response. In the pathophysiology of COVID-19, we can identify disease triggers, mediators, and effector pathways (Fig. 1), which can be targeted by immunotherapy.

Although the disease trigger is infection with SARS-CoV-2, and the first steps of the infection are relatively similar in most patients, the heterogeneity of COVID-19 increases with severity of disease and is largely determined by variability of the host immune response at the level of mediators and effectors. Infection is initiated when the spike glycoprotein of SARS-CoV-2 binds to the human angiotensin-converting enzyme-2 (ACE-2) receptor on the epithelial cell surface, with the host transmembrane protease serine 2 (TMPRSS2) promoting the entry of the virus into the cell ${ }^{1,2}$. ACE2 is highly expressed in the epithelial cells of the nasal cavity, providing a point of entry for SARS-CoV-2 (ref. $\left.{ }^{3}\right)$. The virus is also recognized by pattern-recognition receptors on immune cells, which are responsible for the initiation of the host defense mechanisms. The subsequent production of immune mediators such as cytokines and complement - produced locally in moderate amounts - is essential to fight the infection; however, these can be deleterious when produced in excess 4 .

Several studies have shown that the IL-1-IL-6 axis is likely to represent one of the most biologically relevant signaling pathways

'Department of Internal Medicine and Radboud Center for Infectious Diseases, Radboud University Medical Center, Nijmegen, the Netherlands. ${ }^{2}$ Department of Infectious Diseases, Attikon Hospital, University of Athens, Athens, Greece. ${ }^{3}$ Department of Intensive Care Medicine and Radboud Center for Infectious Diseases, Radboud University Medical Center, Nijmegen, the Netherlands. ${ }^{4}$ Department of Intensive Care, University Medical Center Utrecht, Utrecht, the Netherlands. ${ }^{5}$ Department of Internal Medicine, University Medical Center Utrecht, Utrecht, the Netherlands. ${ }^{6}$ Division of Infectious Diseases, Center for Experimental Molecular Medicine (CEMM), Amsterdam UMC, University of Amsterdam, Amsterdam, the Netherlands. ${ }^{7}$ Department of Intensive Care Medicine and Laboratory of Experimental Intensive Care Medicine and Anesthesiology, Amsterdam UMC, University of Amsterdam, Amsterdam, the Netherlands. ${ }^{8}$ School of Immunobiology and Microbial Sciences, King's College London, London, UK. ${ }^{9}$ Julius Center for Health Sciences and Primary Care, Utrecht, the Netherlands. ${ }^{10}$ UPMC and University of Pittsburgh, Pittsburgh, PA, United States. "Department of Immunology and Metabolism, Life \& Medical Sciences Institute, University of Bonn, Bonn, Germany. ${ }^{\bowtie}$-mail: frank.vandeveerdonk@radboudumc.nl; mihai.netea@radboudumc.nl 


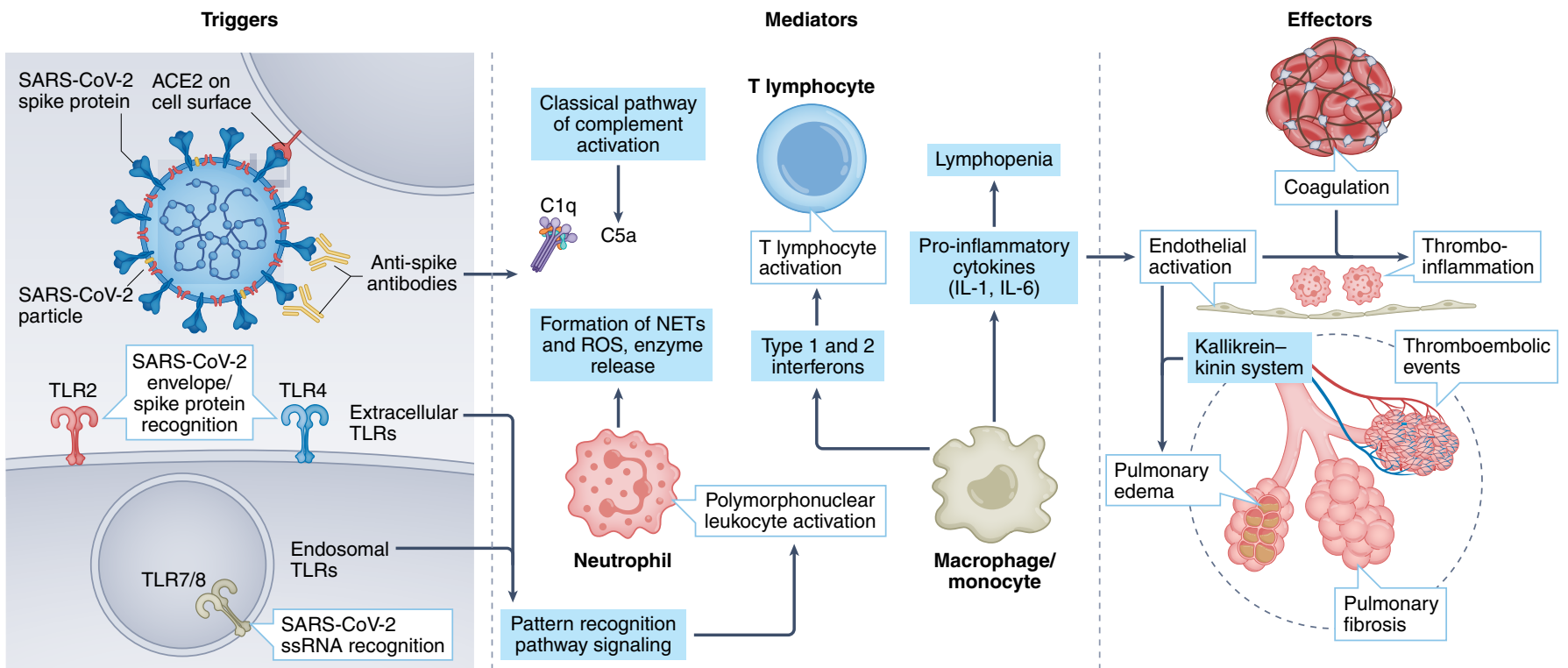

Fig. 1 | Pathophysiological factors targeted by immune-based therapies in COVID-19. A summary of the pathophysiological factors targeted by immune-based therapies in COVID-19, which can be categorized as triggers of the infection (for example, SARS-CoV-2 virus and recognition receptors), mediators of the immune response (such as cytokines and complement), and immune effector mechanisms (the kallikrein-kinin system and thromboinflammation). ACE2, angiotensin converting enzyme 2; IL-1/6, interleukin 1/6; NET, neutrophil extracellular trap; ROS, reactive oxygen species; ssRNA, single-stranded RNA; TLRs, Toll-like receptors.

in the SARS-CoV-2-induced hyperinflammatory reaction ${ }^{5-7}$. Interestingly, in patients with severe COVID-19, low HLA-DR expression on circulating monocytes (a marker of immunosuppression) was clearly evident, but the monocytes retained normal to high production of cytokines (in contrast to bacterial sepsis) ${ }^{5,8}$. At the cellular level, COVID-19 is associated with a marked decrease in circulating $\mathrm{CD}^{+}$and $\mathrm{CD} 8^{+} \mathrm{T}$ cells ${ }^{9}$, reminiscent of sepsis-associated lymphopenia ${ }^{10}$, and this is associated with disease severity and poor outcome $^{11}$. In addition to this reduction in lymphocyte numbers, their function and capacity to release type II interferons is also severely affected in patients with severe COVID-19 (refs. ${ }^{12-15}$ ).

Additional important pathophysiological processes in COVID19 are induced at the level of effector pathways, such as the coagulation system. Thrombi occur when hypercoagulability, endothelial injury and blood stasis converge, and these conditions are frequently encountered in severe COVID-19. Subsequently, arterial and venous thromboembolisms have been frequently reported: studies show that between $21 \%$ and $69 \%$ of patients with severe COVID-19 develop thromboembolic complications ${ }^{16}$. It is believed that inflammatory processes have an important role in the induction of thromboembolic processes, leading to severe complications ${ }^{17-19}$. In later phases, patients may develop pulmonary fibrosis, or they may enter a more chronic phase known as long COVID ${ }^{20}$.

All in all, the pathophysiology of COVID-19 is complex, comprising an interaction between hyperinflammation, defective lymphocyte function, endothelial dysfunction, thromboembolic complications and fibrotic processes in the lung. These processes are not only complex, but are also highly variable between patients, probably related to the heterogeneity of the host immune response. This warrants a stratified immunotherapy approach in clinical trials for COVID-19.

\section{Immunotherapy for COVID-19}

From the start of the COVID-19 pandemic, it became clear that dysregulation of immune responses against SARS-CoV-2 is one of the main features of disease pathogenesis, especially in patients with severe disease, and studies aimed at rebalancing this using modulators of immune responses were initiated early on. Our aim is to provide an overview of the immunotherapies that target different components of COVID-19 pathophysiology, and to propose a practical approach for the use of host-directed strategies in clinical practice. Table 1 provides an overview of the most important clinical trials of immunotherapy in COVID-19.

Anti-virus immunotherapies (anti-trigger). Eliminating the virus as early as possible is likely to prevent or limit the cascade of immune dysregulation and therefore severity of disease. One aspect that is important to mention is that new studies provided important information on antiviral therapy, such as remdesivir and molnupiravir in COVID-19. However, because these are not considered immunomodulatory drugs, we will not focus on their use, but on the studies using immunotherapeutic drugs. Immune-based virus elimination with either polyclonal convalescent plasma or human monoclonal antibodies to the SARS-CoV-2 spike protein might prevent infection in susceptible individuals at risk or might improve outcomes in those who have established COVID-19. The underpinning biology with immunoglobulin therapies is the provision of immediate antiviral humoral immunity that on the one hand reduces the viral load, and on the other hand may induce immunomodulation through Fc gamma receptors ${ }^{21,22}$, with both mechanisms contributing to reduction of illness severity and improved outcomes. It must be noted, however, that the role of Fc gamma receptors remains controversial in COVID-19 pathogenesis, with some literature referring to its role as a disease-enhancing factor ${ }^{23,24}$.

There is relatively solid data for efficacy of convalescent plasa when high-titer plasma is used early in severe infection, with the first data on the use of convalescent plasma in infectious diseases going back to the $1930 \mathrm{~s}^{25}$, and this treatment has been explored in COVID-19 from the very beginning of the pandemic ${ }^{26-28}$. A living systematic review by the Cochrane Collaboration on SARS-CoV-2 convalescent plasma analyzed data from randomized clinical trials that had been conducted as of 20 May 2021 (ref. ${ }^{29}$ ). There was no 


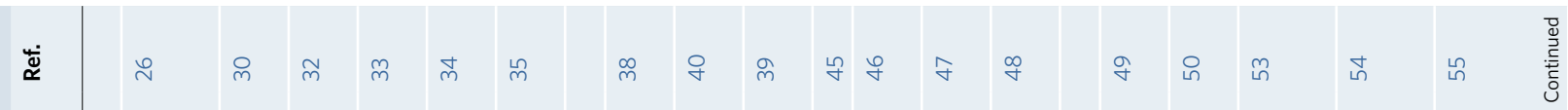

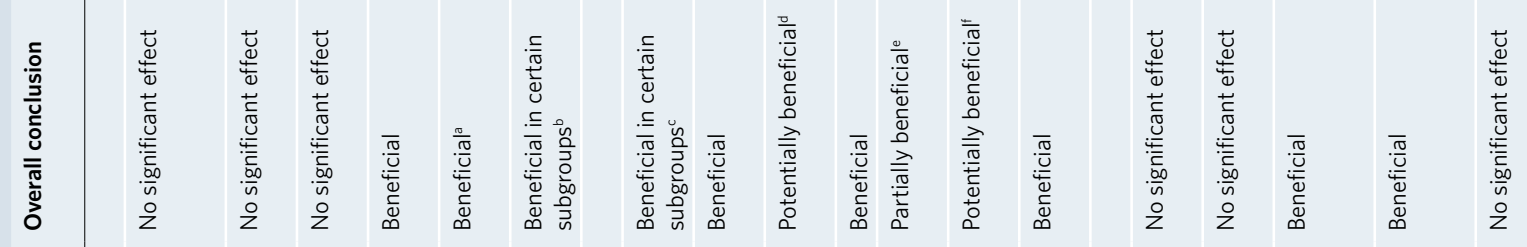

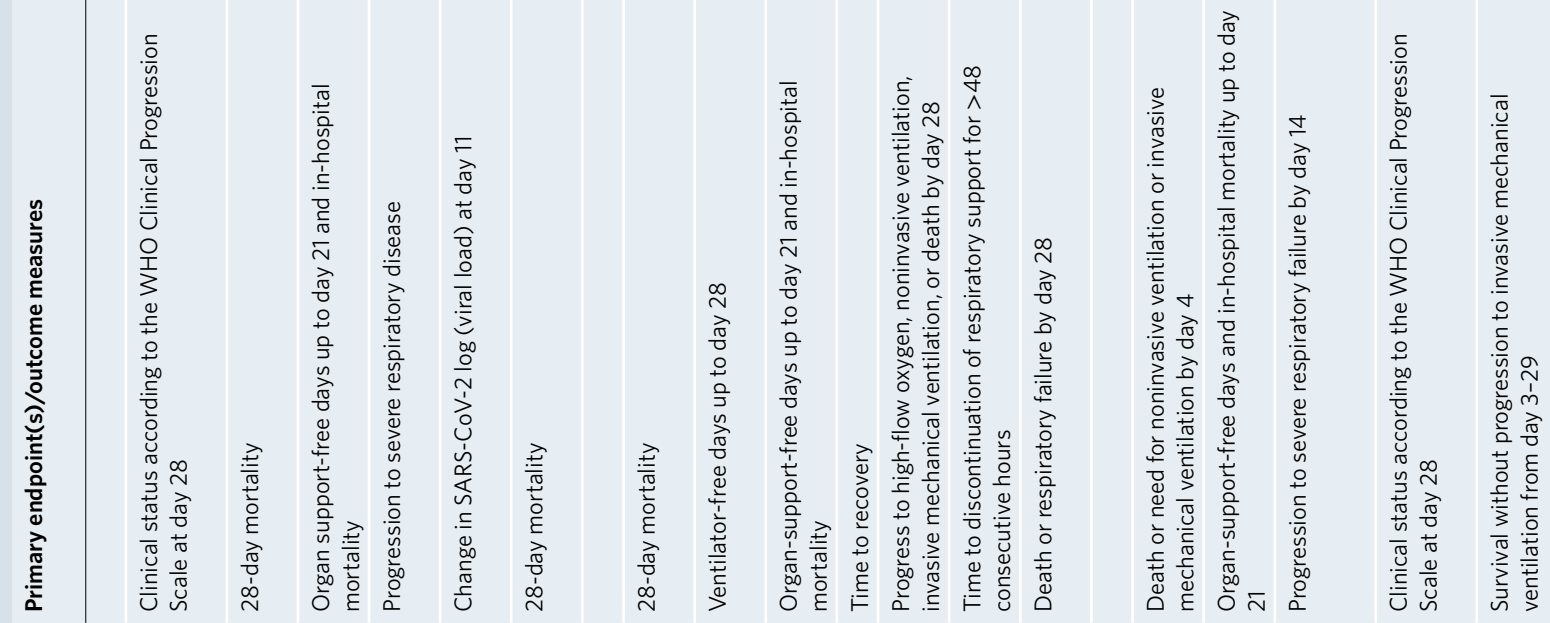
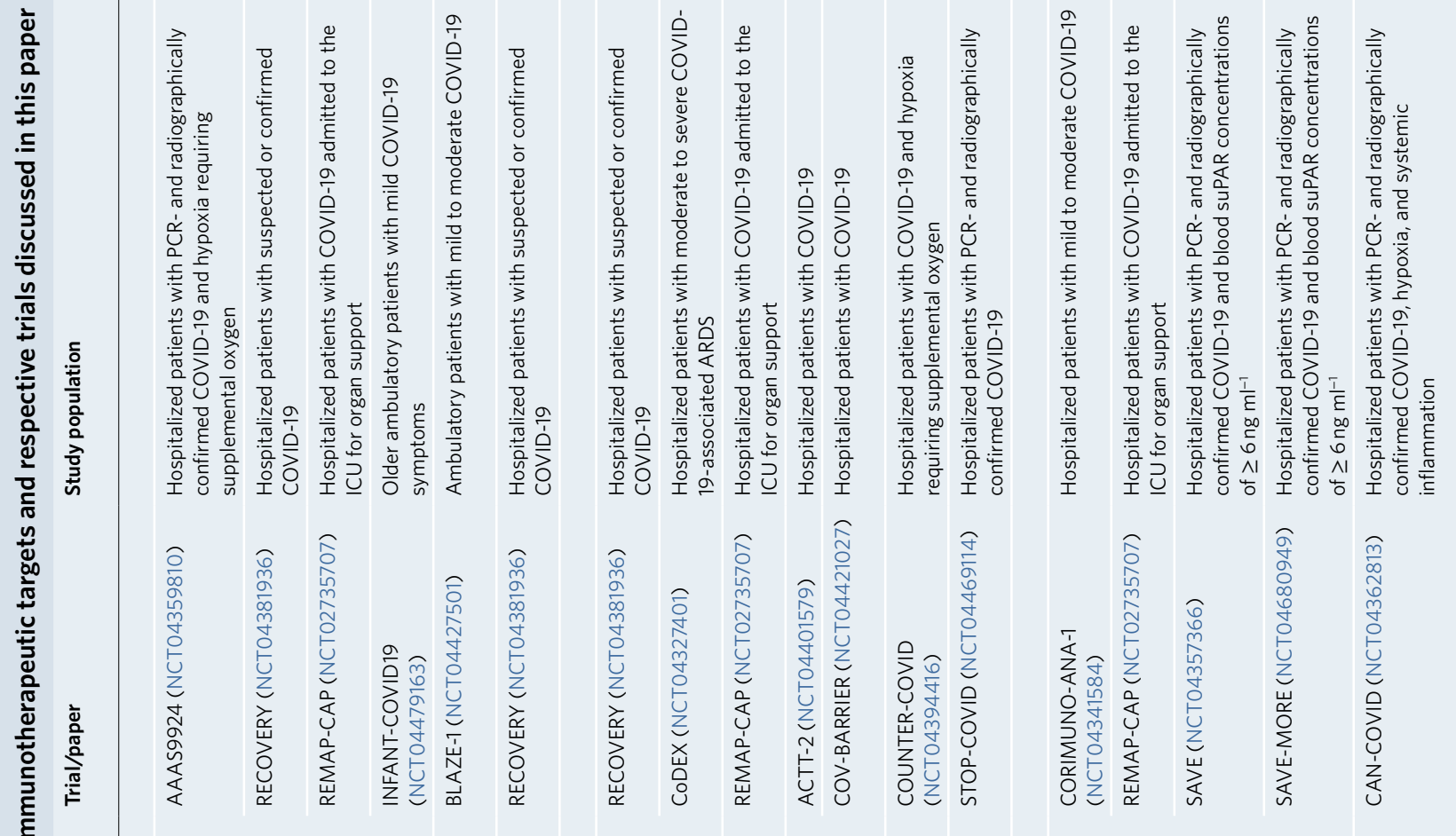

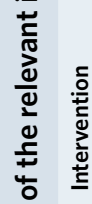
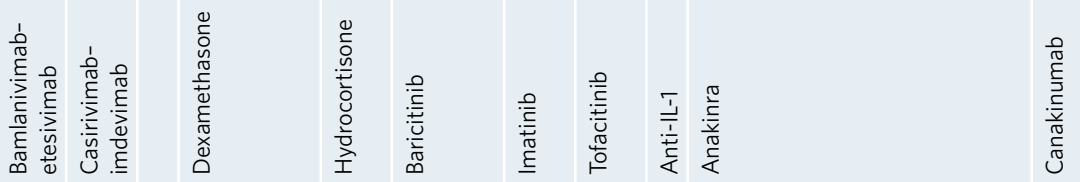

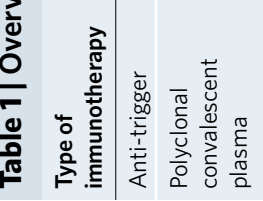
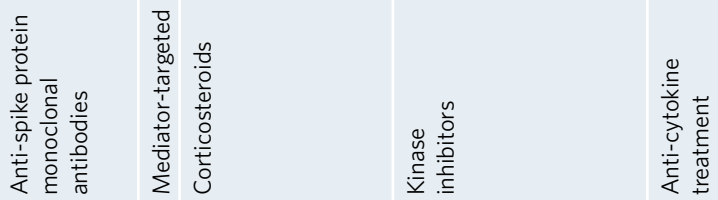


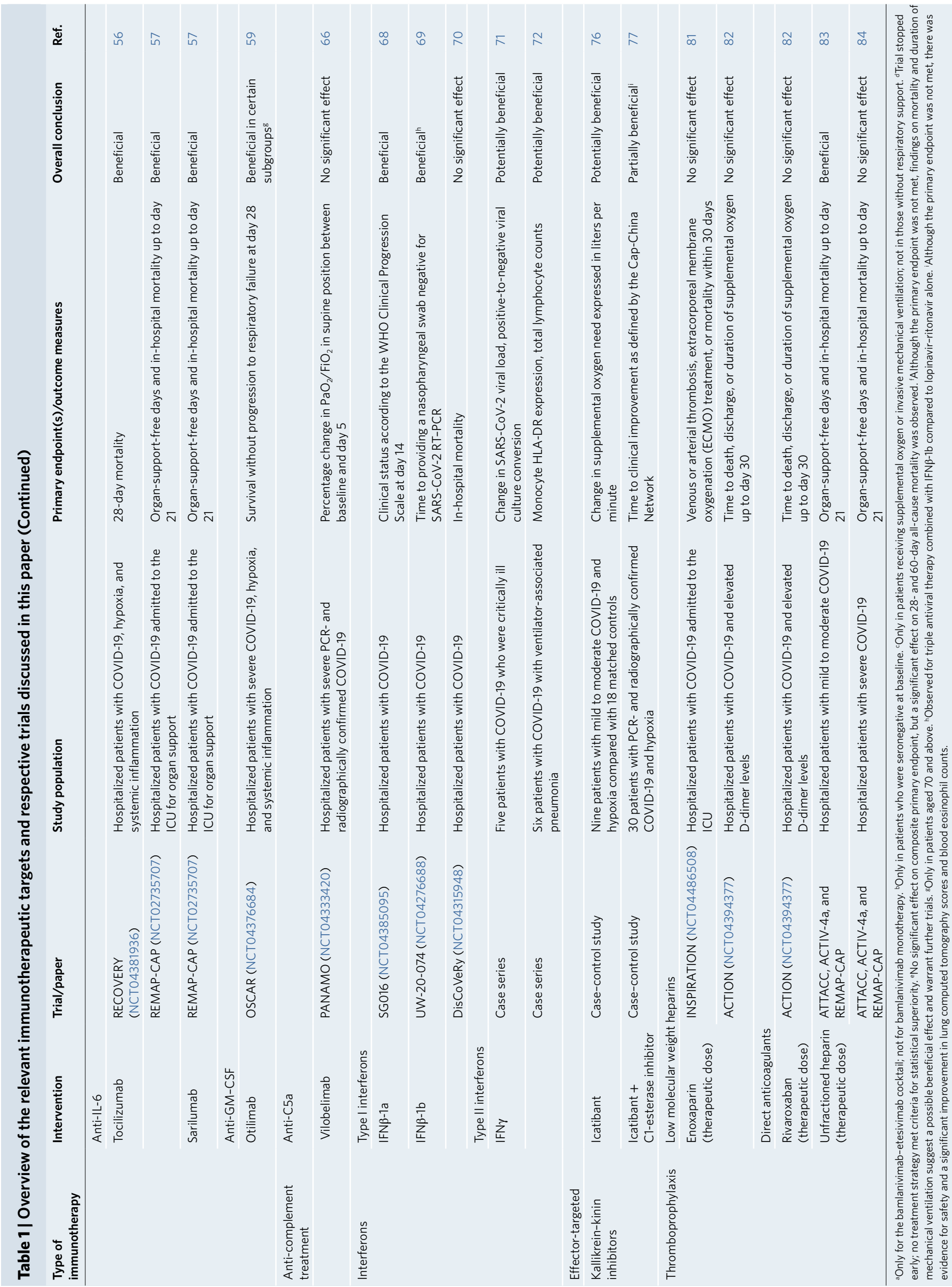


difference in all-cause 28-day mortality (risk ratio (RR) $0.98,95 \%$ confidence interval (CI) $0.92-1.05 ; 7$ randomized control trials (RCTs), 12,646 participants; high-certainty evidence). Similarly, neither the United Kingdom RECOVERY ${ }^{30}$ trial that enrolled mainly ward patients, nor the global REMAP-CAP ${ }^{31}$ trial, in which most patients were mechanically ventilated, showed any benefit for treatment with convalescent plasma. However, in immunocompromised patients and older patients who may be immunosenescent ${ }^{32}$, early administration of convalescent plasma seems to be potentially beneficial, although this is based on smaller trials with fewer patients included ${ }^{33}$.

Another strategy is the use of monoclonal antibodies, which differ from convalescent plasma, because they act against one predefined target, such as the spike protein, with high neutralizing activity. In high-risk ambulatory patients, a combination of bamlanivimab and etesevimab reduced COVID-19-related hospitalizations, reduced viral load and illness duration, and decreased mortality ${ }^{34}$. Another antibody preparation, a combination of the monoclonal antibodies casirivimab and imdevimab (REGEN-COV), reduced 28-day mortality among hospitalized patients who were seronegative at baseline $^{35}$. Antiviral immunotherapy is likely to exert therapeutic potential when given early, especially before the endogenous development of antibodies. Although this treatment may not be of benefit when endogenous antibody production is mounted in later stages of disease, it theoretically may benefit some patients, such as those who are immunocompromised and remain seronegative with persistent detectable viral loads ${ }^{13,36}$.

Immunotherapies targeting immune mediators of host defense. The immune response can also be modulated by targeting the mediators that are triggered by the virus and which drive several effector mechanisms (Fig. 1). These can be non-specific and broad, such as corticosteroids, or very targeted, such as inhibiting one specific cytokine.

Corticosteroids. In a retrospective cohort study of 201 patients admitted with confirmed COVID-19 pneumonia in Wuhan, China, in early 2020, treatment with methylprednisolone was associated with reduced risk of death (hazard ratio, 0.38; 95\% CI, 0.20-0.72) among patients with acute respiratory distress syndrome (ARDS) ${ }^{37}$. However, effectiveness of untargeted immune suppression needed to be demonstrated with high-quality evidence, ideally from randomized studies, to be accepted by the scientific community. To this end, the RECOVERY RCT (an adaptive platform design) was the first to report that dexamethasone $(6 \mathrm{mg}$ once daily for up to 10 days) reduced 28-day mortality in patients hospitalized with COVID-19 (ref. ${ }^{38}$ ). In that study, 2,104 patients were assigned to receive dexamethasone and 4,321 to receive usual care. Overall, 28 -day mortality was $22.9 \%$ in the dexamethasone group and $25.7 \%$ in the control group (age-adjusted rate ratio 0.83 ; 95\% CI, $0.75-$ 0.93). However, reduced incidence of death in the dexamethasone arm was found for those receiving invasive mechanical ventilation (rate ratio, $0.64 ; 95 \% \mathrm{CI}, 0.51-0.81$ ) and those receiving oxygen without invasive mechanical ventilation (rate ratio, $0.82 ; 95 \% \mathrm{CI}$, 0.72-0.94) - in other words, the patients who were sicker at the time of treatment seemed to benefit from corticosteroids. Similar protective effects of steroids in patients with severe COVID-19 were reported in REMAP-CAP, another adaptive platform study, in which 403 patients were included in a corticosteroid evaluation domain ${ }^{39}$. The median adjusted odds ratio and Bayesian probability of superiority for the primary endpoint (combined organ support-free days at 21 days and mortality) were 1.43 (95\% credible interval (CrI), $0.91-2.27)$ and $93 \%$ for fixed-dose hydrocortisone, and $1.22(95 \%$ CrI, $0.76-1.94)$ and $80 \%$ for shock-dependent hydrocortisone, compared with control. Two other large studies from Brazil and France also supported a benefit from corticosteroids in patients with severe

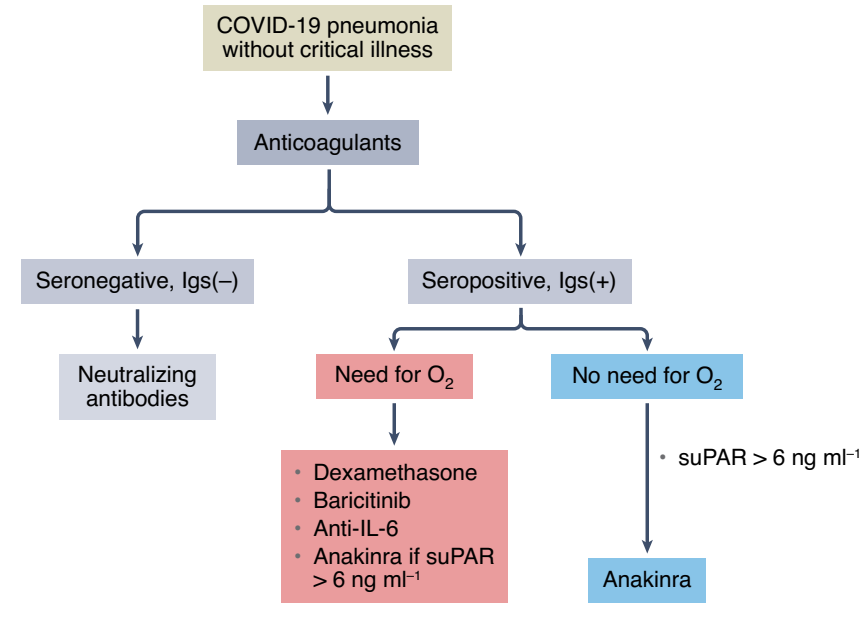

Fig. 2 | Treatment guide for moderate to severe COVID-19, without critical illness. A decision-making chart for immunotherapy of patients with moderate to severe COVID-19, without critical illness. Igs, immunoglobulins; IL-6, interleukin-6; $\mathrm{O}_{2}$, supportive oxygen therapy; suPAR, soluble urokinase plasminogen activator receptor.

COVID-19 (refs. $\left.{ }^{40,41}\right)$. After these results were released, similar corticosteroid trials terminated enrollment and combined their data in a prospective meta-analysis led by the World Health Organization $(\mathrm{WHO})^{42}$, which provides a high level of evidence for the effectiveness of corticosteroids in hospitalized patients with COVID-19 who need respiratory support.

The observation that the beneficial effects of steroids are significant in sicker patients could be explained by the pleiotropic effects of steroids that target different pathophysiological components of COVID-19 present in severe disease. Although this might explain why so many patients benefit, it also makes it challenging to define who needs to be treated with corticosteroids when progressing towards severe disease (Fig. 2). Another important consideration is the possible over-use of corticosteroids, especially in the early phase of disease when such treatment might lead to detrimental effects, further supporting the need for guidance of immunotherapy.

Kinase inhibitors. Tyrosine kinases also have pleiotropic effects and are seen as attractive targets in treating COVID-19, given their established druggability and the fact that most tyrosine kinase inhibitors have a well-known clinical safety profile ${ }^{43,44}$. Tyrosine kinase inhibitors can block cytokine signaling pathways and many immune effector pathways.

A double-blind, randomized, placebo-controlled trial of 1,033 adults hospitalized with COVID-19 who were randomly assigned to receive oral baricitinib (a Janus tyrosine kinase (JAK) inhibitor) or placebo for up to 14 days demonstrated that patients receiving baricitinib had a shorter time to recovery than patients in the placebo group (median 7 versus 8 days) ${ }^{45}$. Importantly, the effect was more pronounced in the subgroup that required high-flow oxygen or noninvasive ventilation when compared with those who received placebo (10 versus 18 days). In a phase 3, double-blind, randomized, placebo-controlled trial with 1,525 participants, 764 received baricitinib and 76 received placebo ${ }^{46}$. There was a $38.2 \%$ relative reduction in mortality, with the 28 -day all-cause mortality being $8 \%$ for baricitinib and $13 \%$ for placebo with a hazard ratio of 0.57 (95\% CI 0.41-0.78). This was an additional effect to standard treatment, including corticosteroids, since $79.3 \%$ of participants with available data received systemic corticosteroids at baseline. The FDA has recently authorized baracitinib for emergency use to treat COVID-19. A Dutch clinical trial of 400 hospitalized patients with COVID-19 found a beneficial effect of oral imatinib 
(a cytosolic multi-tyrosine kinase inhibitor) compared with placebo on duration of mechanical ventilation (7 days versus 12 days) and 28 -day mortality ( $8 \%$ versus $14 \%)^{47}$. It should be noted that the primary endpoint was not met, which was time to discontinuation of mechanical ventilation and supplemental oxygen for more than 48 consecutive hours while being alive during a 28 -day period. However, the beneficial findings warrant follow-up trials to validate these outcomes and select which patients might benefit from treatment with imatinib. Other kinase inhibitors under investigation in RCTs in hospitalized patients with COVID-19 include those targeting Bruton's tyrosine kinases (for example, ibrutinib, acalabrutinib and zanubrutinib), phosphatidylinositol-3-kinase (PI3K)/ mammalian target of rapamycin (mTOR) inhibitors (duvelisib and temsirolimus), and JAK inhibitors (such as ruxolitinib and tofacitinib) ${ }^{44}$. Recently, in a trial in Brazil, 289 patients hospitalized with COVID-19 were randomized to receive tofacitinib or placebo. They showed a cumulative incidence of death or respiratory failure of $18.1 \%$ in the tofacitinib group and $29.0 \%$ in the placebo group (risk ratio, $0.63 ; 95 \%$ CI $0.41-0.97 ; P=0.04$ ) at day 28 (ref. ${ }^{48}$ ). Therefore, there is a good rationale to explore tyrosine kinase inhibitors as a COVID-19 therapy, and the results reported thus far encourage further exploration in larger trials.

Targeted strategies: anti-cytokine treatment. Both IL-1 and IL-6 induce local effects, such as macrophage activation, endothelial leakage and liquid extravasation, as well as systemic effects including fever, somnolence and synthesis of acute-phase proteins. Although moderate induction of inflammation is necessary for host defense, overabundant release of these mediators is deleterious. The CORIMUNO-ANA study randomized 116 patients with mild to moderate COVID-19 pneumonia to treatment with placebo or the IL-1 inhibitor anakinra, the only immunological criterion being a plasma C-reactive protein (CRP) level higher than $25 \mathrm{mg} \mathrm{l}^{-1}$. No significant effect of blocking IL-1 with anakinra was observed on the proportion of patients who died or needed noninvasive or mechanical ventilation at day 4 , or on survival without need for mechanical or noninvasive ventilation at day 14 (ref. ${ }^{49}$ ). In line with this, anakinra had no effect on survival or release from organ support in the REMAP-CAP trial, in which 378 patients with COVID-19 needing organ support (without further immunological stratification) in the intensive-care unit (ICU) were treated with anakinra and compared with 418 controls $^{50}$.

By contrast, patient stratification based on immunological profiles did identify patients likely to benefit from IL-1 blockade. The soluble urokinase plasminogen receptor (suPAR) was found to be associated with the risk for progression into severe respiratory failure, and this formed the basis of a biomarker-driven immunotherapy trial ${ }^{51,52}$ (Box 1). In the open-label single-arm phase 2 SAVE study, 130 patients with COVID-19 pneumonia and plasma suPAR of $6 \mathrm{ng} \mathrm{ml}^{-1}$ or more received SoC treatment and anakinra $(100 \mathrm{mg}$ subcutaneously), which blocks both IL- $1 \alpha$ and IL-1 $\beta$, daily for 10 days. The incidence of severe respiratory failure and/or death after 14 days was $22.3 \%$ compared with $59.2 \%$ of matched patients receiving SoC alone ${ }^{53}$.

These results provided the rationale for the double-blind randomized phase 3 SAVE-MORE trial, in which 594 patients with moderate to severe COVID-19 pneumonia (WHO scale $3-5$ ) and suPAR of $6 \mathrm{ng} \mathrm{ml}^{-1}$ or more were randomized to treatment with SoC and placebo $(n=189)$ or SoC and anakinra $(n=405)$. Anakinra treatment provided 2.78 times higher odds for clinical improvement based on the 11-point WHO Clinical Progression Scale towards both full resolution and critical illness or death after 28 days $^{54}$. The 28-day mortality was lower among patients allocated to anakinra treatment $-6.9 \%$ in the control group versus $3.2 \%$ treated with anakinra. Overall, $85.9 \%$ of patients were co-administered dexamethasone, but anakinra still improved outcomes in this context.
Box 1. The role of biomarker-driven immunotherapy in COVID-19

The emergence of SARS-CoV-2 infection has been associated with a flurry of studies investigating biomarkers associated with disease severity and outcome. Many inflammatory biomarkers, from the number of subpopulations of immune cells (for example, lymphopenia and the neutrophil-to-lymphocyte ratio), to circulating cytokines (such as IL-6 and chemokines) or acute-phase proteins (such as CRP and ferritin), to biomarkers of endothelial cell activation (such as suPAR) or complement, are associated with development of severe COVID-19. Unfortunately, a large gap persists between the use of these biomarkers for predicting disease severity and for patient stratification to improve host-directed (immune-based) therapies. In addition, more work needs to be done to understand the variability of various immunological biomarkers in time, which may also influence treatment approaches. Although the readily available anti-COVID-19 immunotherapies (such as steroids and anti-IL-6 therapies) have already been explored, the next steps for optimizing immunotherapy will require identification of patient subgroups that would benefit from specific approaches: for example, immune-modulating approaches in patients with hyperinflammation versus immune-stimulatory therapies in those with immune paralysis. A blueprint for biomarker-guided therapies is provided by the use of suPAR to guide anakinra treatment in the subgroup of patients with COVID-19 with lung hyperinflammation $^{54}$, or the use of HLA-DR expression to guide IFN $\gamma$ treatment ${ }^{106}$. Intense biomarker research focusing on patient stratification is warranted; in addition, biomarkers to enable the monitoring of the effects of immune-based therapies are also needed.

The results of the SAVE-MORE trial suggest that anakinra treatment guided by suPAR is a therapeutic strategy before progression into critical illness.

A trial including 454 patients randomized 1:1 to placebo or canakinumab, which blocks only IL- $1 \beta$, did not reach significance for its primary outcome, which was survival without invasive mechanical ventilation at day 29 (ref. ${ }^{55}$ ). Patients enrolled were hypoxic and hospitalized without the need for invasive mechanical ventilation. COVID-19-related mortality occurred in 11 out of 223 patients (4.9\%) in the canakinumab group versus 16 out of 222 (7.2\%) in the placebo group, with a rate difference of $-2.3 \%(95 \%$ $\mathrm{CI},-6.7 \%$ to $2.2 \%)$ and an odds ratio of 0.67 (95\% CI, $0.30-1.50)$.

For patients with hypoxemia and in need of oxygen therapy, anti-IL-6 strategies have been shown to be beneficial by the large-scale platforms RECOVERY ${ }^{56}$ and REMAP-CAP ${ }^{57}$. In the open-label, randomized RECOVERY trial, which predominantly included patients who were not critically ill, 2,094 patients received usual care, and 2,022 patients received the IL- 6 inhibitor tocilizumab. Mortality was decreased from $35 \%$ in the usual care arm to $31 \%$ in the tocilizumab arm $(P=0.0028)^{56}$. The REMAP-CAP trial included 2,274 critically ill participants, with 972 participants receiving tocilizumab, 485 randomized to sarilumab, 378 to anakinra, and 418 to control. Tocilizumab and sarilumab were both effective, when compared with control, and likely to be equivalent in terms of improving survival and release from organ support. However, anakinra was not effective in this population. Median organ-support-free days were 7 (interquartile range (IQR) -1, 16), 9 (IQR -1, 17), 0 (IQR -1, 15) and 0 (IQR -1, 15) for tocilizumab, sarilumab, anakinra, and control, respectively. Median adjusted odds ratios for hospital survival were 1.42 (95\% CrI 1.05, 1.93), 
$1.51(95 \%$ CrI 1.06, 2.20) and 0.97 (95\% CrI 0.66, 1.40) for tocilizumab, sarilumab, and anakinra, respectively, compared with control $^{57}$. The WHO Rapid Evidence Appraisal for COVID-19 Therapies (REACT) Working Group published a prospective meta-analysis of clinical trials of patients hospitalized for COVID-19 that showed an association with lower 28-day all-cause mortality in patients treated with IL-6 antagonists compared with patients that received usual care or placebo ${ }^{58}$. Collectively, these data support the use of blocking IL-6 in patients with COVID-19 who are hospitalized and in need of oxygen supplementation.

Other proinflammatory cytokines besides those in the IL-1-IL-6 axis are also involved in COVID-19-mediated inflammation; one attractive approach is to inhibit neutrophil recruitment in the lung through inhibition of GM-CSF. In the double-blind randomized trial OSCAR, patients with respiratory distress were randomized to receive one infusion of the monoclonal anti-GM-CSF otilimab $(n=395)$ or placebo $(n=398)$. The primary study endpoint was the rate of patients being alive and free of respiratory failure by day 28 : this was $71 \%$ in the placebo group and $67 \%$ in the otilimab group $(P=0.09)$. However, in the group of patients aged 70 years or older, there was a significant effect of otilimab on the primary endpoint, namely $66 \%$ in the placebo compared with $46 \%$ in the group that received otilimab $(P=0.009)^{59}$, which provides the rationale to further explore otilimab in patients aged 70 years or more. Nonetheless, one should be cautious with such age-dependent interpretations, as they may imply opposite negative effects in the younger patients. Other cytokine-targeted therapies, such as anti-TNF, are currently being studied (NCT04705844). Cytokine-targeted treatment strategies for COVID-19 seem to be an attractive approach and might benefit from biomarker-based precision RCTs that help identify which patients are likely to benefit the most.

Anti-complement therapies: anti-C5a. Complement activation seems to contribute to the pathophysiology of severe COVID-19. Autopsies of patients with severe COVID-19 showed widespread complement activation in the lung and kidney ${ }^{60,61}$. The potent anaphylatoxin $\mathrm{C} 5 \mathrm{a}$ increases adherence and migration of neutrophils and monocytes to blood vessel walls; this causes tissue damage by oxidative radical formation and enzyme release, but also induces release of tissue factor from endothelial cells and neutrophils, thereby activating the coagulation system ${ }^{62-64}$. In patients with severe COVID-19, high concentrations of C5a are associated with poor outcomes ${ }^{65}$. On the basis of these observations, anti-complement therapies have been investigated in severe COVID-19. One randomized phase 2 open-label trial $(n=30)$ investigated blockade of C5a using a chimeric monoclonal IgG4 antibody (vilobelimab) that specifically binds with high affinity to the soluble form of human C5a and was shown to be safe in patients with severe COVID-19. In this study, infections considered as serious adverse events were reported in three (20\%) patients receiving direct C5a inhibition, versus five (33\%) patients in the control group ${ }^{66}$. The secondary outcomes, including severe pulmonary embolism and mortality, were in favor of anti-C5a treatment. A phase 3 trial (NCT04333420) that aims to enroll 360 patients with severe COVID-19 and use28-day mortality as the primary endpoint is ongoing.

Stimulators of antiviral defense: interferons. Type I IFNs are crucial for antiviral host responses, and they have previously been used with partial success against severe acute respiratory syndrome (SARS) ${ }^{67}$. Daily inhalations with IFN $\beta$-1a for 14 days versus placebo was investigated in a double-blind RCT in 101 patients with COVID19 in the United Kingdom. Patients receiving inhaled IFN $\beta$-1a had greater odds of improvement (odds ratio 2.32; 95\% CI 1.07-5.04; $P=0.033$ ) on day 15 or 16 , and were more likely to recover during treatment (hazard ratio $2.19 ; 95 \%$ CI $1.03-4.69 ; P=0.043$ ) ${ }^{68}$. In a multicenter, prospective, open-label, randomized, phase 2 trial in
China, 127 patients received either triple antiviral therapy (lopinavir, ritonavir and ribavirin) and 3 doses of 8 million international units of IFN $\beta$-1b on alternate days $(n=86)$ or lopinavir and ritonavir $(n=41)$. Again, triple antiviral therapy plus IFN $\beta-1$ b resulted in shorter viral shedding and faster clinical improvement compared to lopinavir-ritonavir alone in patients with mild to moderate COVID-19 (ref. ${ }^{69}$ ). By contrast, in the WHO Solidarity trial, in which IFN $\beta$-1a was given subcutaneously and intravenously for 6 days, death occurred in 243 out of 2,050 patients receiving IFN $\beta$-1a and in 216 out of 2,050 receiving its control (rate ratio, 1.16; $95 \% \mathrm{CI}$, $0.96-1.39 ; P=0.11)^{70}$. An important note is that half of the patients in the Solidarity trial received corticosteroids that might affect interferon signaling, but the clinical relevance of this is uncertain.

Interferon-gamma (IFN $\gamma$ ) is a type II interferon that has an important role in boosting the innate host defense and might therefore act as an immunostimulatory agent. In a case series of five patients with persistent high viral loads and poor clinical condition with secondary infectious complications, recombinant IFN $\gamma$ showed viral culture conversion from positive to negative and rapid decrease in viral load by PCR without subsequent signs of hyperinflammation ${ }^{71}$. In another report with six non-immunocompromised patients with ventilator-associated pneumonia, IFN $\gamma$ treatment led to a fast increase in HLA-DR ${ }^{\text {hi }}$ monocytes in all but one patient, and was well tolerated ${ }^{72}$. IFN $\gamma$ might represent an immunostimulatory agent that could help to clear viral infection and be beneficial in the setting of secondary infections in critically ill patients with COVID-19. Other strategies to boost the immune system are checkpoint inhibitors or recombinant IL-7, and these are currently under investigation (NCT04335305, NCT04379076) . $^{73}$.

Immunotherapies that target effector pathways. Inhibitors of local pulmonary edema: kallikrein-kinin system. Timely inhibition of the kallikrein-kinin system in patients with COVID-19 is proposed to counteract pulmonary edema and suppress thromboinflammation $^{74,75}$, thereby limiting disease severity. In a case-control study of 9 patients with COVID-19 treated with icatibant (bradykinin B2 receptor antagonist) and 18 matched controls, icatibant showed promising results compared to SoC treatment ${ }^{76}$. Directly after treatment with 3 doses of $30 \mathrm{mg}$ of icatibant, a reduction in oxygen supplementation of $31 \mathrm{~min}^{-1}$ or greater was observed in $89 \%$ of patients in the intervention group compared with $17 \%$ of patients in the control group. Another case-control study investigating the effects of icatibant and an inhibitor of C1-esterase/kallikrein in 30 patients found no significant effect on clinical outcome, but found that both drugs were safe and had beneficial effects on lung CT severity scores and blood eosinophil counts ${ }^{77}$. Disease severity and timing of treatment may be important factors determining the efficacy of icatibant as a COVID-19 treatment. Several other drugs that modulate the kallikrein-kinin system are currently under investigation.

Modulation of immune-thrombotic complications. Damage to the vascular endothelium induced by the inflammatory reaction, together with activation of platelets and the coagulation system, are key pathophysiological features of COVID-19 (refs. ${ }^{78,79}$ ). These host response aberrations have been implicated in the high occurrence of venous thromboembolic disease or arterial thrombosis in COVID19 despite conventional thromboprophylaxis ${ }^{80}$. Consequently, many clinicians and scientific societies proposed the use of thromboprophylaxis medication at higher doses than usual in clinical practice, and over 75 RCTs related to antithrombotic therapy in hospitalized patients with COVID-19 have been initiated ${ }^{79}$.

In a multicenter RCT conducted in Iran, encompassing 562 ICU patients with COVID-19, intermediate-dose prophylactic anticoagulation (enoxaparin $1 \mathrm{mg} \mathrm{kg}^{-1}$ ) compared with standard-dose prophylactic anticoagulation (enoxaparin $40 \mathrm{mg}$ ) did not impact the primary outcome, which was a composite of venous or arterial 
thrombosis, treatment with extracorporeal membrane oxygenation, or mortality ${ }^{81}$. In a multicenter RCT in Brazil in 615 hospitalized patients, of whom $94 \%$ were considered clinically stable, anticoagulation with rivaroxaban or enoxaparin followed by rivaroxaban to day 30 did not improve clinical outcomes and increased bleeding compared with prophylactic anticoagulation ${ }^{82}$.

By contrast, two open-label adaptive multiplatform RCTs evaluating the use of therapeutic-dose anticoagulation with heparin in hospitalized non-critically ill ${ }^{83}$ and critically ill patients with COVID-19 (ref. ${ }^{84}$ ) were performed. The primary outcome of these RCTs was organ-support-free days, an ordinal scale composed of survival to hospital discharge and-in survivors-the number of days free of organ support to day 21. Among the 2,219 non-critically ill patients, the probability that therapeutic anticoagulation increased organ-support-free days compared with standard thromboprophylaxis was $98.6 \%$ (adjusted odds ratio 1.27 , $95 \%$ CrI 1.03-1.58) ${ }^{83}$. Major bleeding occurred in $1.9 \%$ of patients treated with therapeutic heparin and $0.9 \%$ of patients treated with standard thromboprophylaxis ${ }^{83}$. By contrast, in critically ill patients $(n=1,098)$ therapeutic anticoagulation with heparin did not improve survival or days free of organ support ${ }^{84}$. Major bleeding occurred in $3.8 \%$ of patients assigned to therapeutic anticoagulation versus $2.3 \%$ of patients on standard thromboprophylaxis ${ }^{84}$.

Collectively, the results of these first RCTs suggest that therapeutic dose heparin might be beneficial in hospitalized non-ICU patients with COVID-19, whereas therapeutic dose oral anticoagulants are not. In addition, therapeutic dose heparin does not improve the outcome of critically ill patients with COVID-19 and likely is associated with harm. A mechanistic explanation for these observations is currently not known and the results are counterintuitive from the coagulation point of view. This is most likely due to the use of a pleiotropic drug (heparin) in a heterogeneous disease (COVID-19), underscoring the importance of patient stratification-that is, precision medicine. It is tempting to speculate that these differences are explained by heterologous effects on immune effector pathways, but this remains to be demonstrated. Other antithrombic drugs under investigation in RCTs in hospitalized patients with COVID-19 include tissue type plasminogen activator (a profibrinolytic agent), several antiplatelet drugs (dipyramidole, aspirin, clopidogrel) and nafamostat (a serine protease inhibitor and a short-acting anticoagulant). Moreover, several trials have been initiated to evaluate the effect of thromboprophylaxis in patients with COVID-19 following hospital discharge ${ }^{79}$.

Antifibrotic therapies in COVID-19. Development of fibrosis may be related to organizing pneumonia following acute lung injury or the abnormal immune response in the lung, as pulmonary compartmentalization of hyperinflammation is present in patients with COVID-19 (ref. ${ }^{4,85}$ ). It is not known why some may recover from this insult, whereas others respond with an unchecked cellular proliferation, including accumulation of fibroblasts and myofibroblasts, and deposition of collagen to result in pulmonary fibrosis. For these latter patients with COVID-19, available antifibrotic therapies may be beneficial. Apart from steroids, new compounds, mainly tyrosine kinase inhibitors, have demonstrated efficacy in patients with idiopathic pulmonary fibrosis ${ }^{86,87}$. In addition, preclinical data suggest beneficial effects of inhibitors of Janus kinase-signal transducer and activator of transcription (JAK-STAT) in preventing pulmonary fibrosis ${ }^{88}$. However, there are currently no data on antifibrotic treatment in COVID-19 and severalclinical trials are ongoing.

\section{The immunotherapeutic approach in the clinic}

The large number of trials performed since the beginning of the pandemic have provided an unprecedented amount of knowledge for a disease that has been known for such a short time, but this also raises the challenge of discerning the best path for a systematic and rational treatment of the patient with COVID-19. The first important step in approaching the patient with COVID-19 is to determine the severity of the disease, which is one of the most important criteria for patient stratification. Many clinical trials have used the criterion of severity when investigating different approaches of immunotherapy in hospitalized patients outside of the ICU (patients with moderate to severe disease), or in the ICU (severe to critically ill patients). It is important to note that immunotherapy in COVID-19 is dynamic and in constant development. Therefore, we aim to provide guidance on immunotherapeutic strategies that are supported in expert guidelines, such as anti-IL-6R blockade and corticosteroids; however, we will augment this guidance with possible treatment options for when patients fail to respond and there is a clear clinical rationale for an alternative therapy, even if not yet formally tested in large RCTs.

The patient with moderate disease at high risk of worsening. From the perspective of patient stratification based on severity, the first major group of patients with COVID-19 are those with moderate disease admitted to general medicine wards. The aim of immunotherapy in these patients would be to prevent worsening of the disease, and potentially reducing the duration of hospitalization. The patients with mild disease that do not need hospitalization are believed to be able to recover without the need of immunotherapy, and no studies have been conducted on host-directed therapy in this subgroup.

For patients admitted to general medicine wards, several immunotherapeutic approaches have been proposed (Fig. 2). First, the data available on anticoagulant therapy suggest that therapeutic-dose heparin might be beneficial in these non-ICU patients with COVID-19 (but not patients in the ICU). Second, the serological status of the patient should be assessed; if the patient is seronegative, passive immunization with antibody cocktails should be considered. Third, if the patients are seropositive and the addition of antibody cocktails is not expected to be useful, additional steps need to be taken if the patient displays signs of worsening. If the patient needs oxygen supplementation, treatment with dexamethasone should be initiated. Moreover, the initiation of anti-IL-6 therapy (tocilizumab, sarilumab) is advised if the patient needs oxygen therapy and CRP is higher than 50 (this limit differs in the guidelines of various countries). Furthermore, treatment with the kinase inhibitor baricitinib has been shown to improve outcome in patients with high-flow oxygen therapy and noninvasive ventilation $^{45}$. If the patient does not need oxygen therapy but biomarkers indicate worsening inflammation - for example, suPAR higher than $6 \mathrm{ng} \mathrm{ml}^{-1}$, or the presence of the surrogate markers CRP (more than $50 \mathrm{mg} \mathrm{l}^{-1}$ ) and ferritin (higher than $700 \mathrm{mg} \mathrm{l}^{-1}$ ) — then administration of the IL-1 receptor blocker anakinra should be considered ${ }^{54}$.

The ICU patient. Monoclonal antibodies against COVID-19 are a possible option in patients that have no seroconversion during infection or after vaccination. Treatment with corticosteroids and anti-IL- 6 should be initiated within 48 hours of admission to the ICU. When a patient is transferred from the ward and has not yet received dexamethasone or tocilizumab, it is still an option to start corticosteroids and anti-IL-6 treatment.

Difficult therapeutic decisions on patients with severe COVID19 may need to be made if severe complications develop during the ICU stay. When signs of immunoparalysis are present, reflected by lymphopenia, low HLA-DR expression on monocytes, opportunistic infections (for example, aspergillosis or herpes infections) or a persistent high SARS-CoV-2 load, then stimulatory immunotherapy would be a rational step-but this has not been formally tested in RCTs. From a pathophysiological point of view, and based on small case-series, one might consider immunostimulatory treatments such as recombinant IFN $\gamma$. Similar approaches boosting 


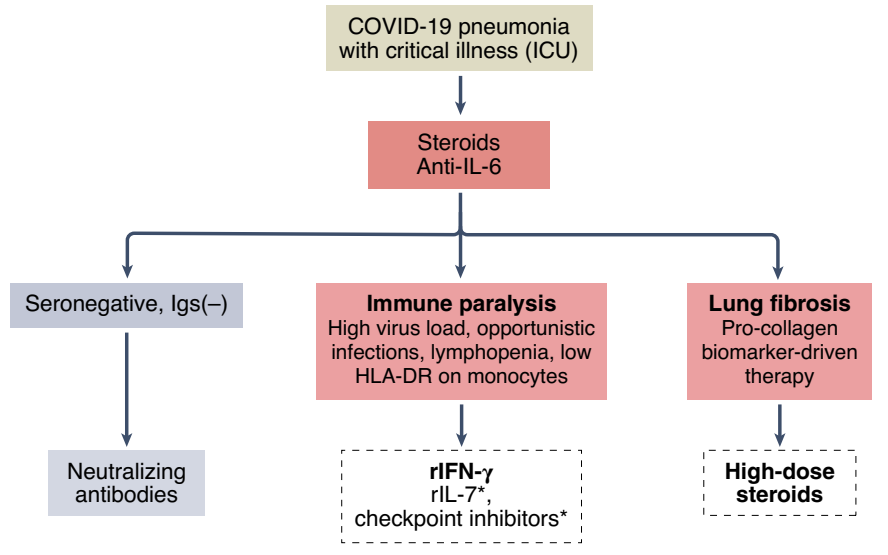

Fig. 3 | Treatment guide for critical illness due to COVID-19. A decisionmaking chart for immunotherapy of patients with critical illness due to COVID-19. Solid boxes depict treatment advice supported by phase 3 clinical trials, whereas boxes in dashed lines depict treatments suggested on the basis of observational and case-series studies alone. Asterisks depict treatments that could be considered, but have not been yet tested. HLA-DR, human leukocyte antigen DR-isotype; ICU, intensive care unit; IL-6, interleukin-6; Igs, immunoglobulins; rIFNy, recombinant interferon-gamma; rlL-7, recombinant interleukin-7.

adaptive immune responses are currently under investigation in clinical trials, such as with recombinant IL-7 and checkpoint inhibitors. Targeting pulmonary fibrosis is another challenge and might benefit from biomarker-directed therapy, although there are currently no data on this in COVID-19. High-dose steroids have been proposed, with evidence coming from trials in the ICU before the pandemic. An overview of the potential approach to immunotherapy in the ICU patient with COVID-19 is presented in Fig. 3.

The patient with multisystem inflammatory syndrome: MIS-C and MIS-A. Early in the pandemic, children were seen to present with diverse COVID-19 symptoms, such as persistent fever, headache, fatigue, abdominal pain, vomiting, conjunctival injection, myocarditis and rash, usually 2-6 weeks after mild COVID-19; this condition was named multisystem inflammatory syndromes in children (MIS-C). A similar syndrome has been described in adults (MIS-A). Some of the children with MIS-C developed multiorgan failure and shock or coronary aneurysms.

The American College of Rheumatology treatment guideline recommends intravenous immunoglobulin (IVIG) and/or high-dose glucocorticoids as first-line therapies in MIS-C ${ }^{89}$. Approximately $30-80 \%$ of patients do not respond to IVIG and may require adjunctive immunomodulatory therapy ${ }^{90-95}$. Pulse methylprednisolone, additional dosing of IVIG, anakinra, tocilizumab and infliximab have all been used as escalation therapies ${ }^{92,96-99}$ in MIS-C. Far fewer cases of MIS-A have been reported in the literature ${ }^{100-102}$. These adult patients were treated with glucocorticoids, with or without IVIG, and anticoagulants with mostly favorable outcomes.

In two large observational cohort studies, the effects of different treatment strategies on short-term outcome were compared, with propensity score adjustments for confounding. The Overcoming COVID consortium reported a lower risk of cardiovascular dysfunction and a lower need for vasopressors and adjunctive therapy in initial treatment with IVIG plus glucocorticoids compared with IVIG monotherapy ${ }^{103}$. Yet, in the Best Available Treatment Study (BATS), treatment with IVIG, IVIG plus glucocorticoids, or glucocorticoid monotherapy did not yield statistically significant differences for endpoints of ventilation, inotropic support or death, or for improvement on an ordinal clinical severity scale ${ }^{104}$. Both studies reported reduced risks for escalation therapy in patients treated with IVIG plus glucocorticoids compared with IVIG monotherapy, which corroborates the findings from a smaller French study ${ }^{105}$; however, glucocorticoid monotherapy and IVIG monotherapy were equally effective.

Differences in study results could result from genetic differences between study populations, differences in viral-strain-dependent hyperimmune responses, and, of course, suboptimal adjustments for all potential confounders, in particular confounding by indication. Therefore, randomized controlled trials are needed to determine the optimal therapy for MIS-C and MIS-A. At present, there is one recruiting RCT comparing infliximab, glucocorticoids or anakinra as escalation therapy after IVIG monotherapy (NCT04898231). In addition, treatment with mesenchymal stromal cells is currently being evaluated in open-label studies (NCT04549285, NCT04456439).

\section{Future outlook and conclusions}

The immunotherapy of COVID-19 has reached important milestones, being the first severe acute infectious disease in which a strong level of evidence permits recommendation of immunotherapy, as detailed above. However, major quandaries remain in the day-to-day clinical practice, and they should be addressed as a matter of urgency.

One major quandary with which we are confronted is the treatment of the patient with COVID-19 who does not improve, despite treatment with immunotherapeutic agents such as dexamethasone and anti-IL- 6 therapy. Some of these patients remain strongly hyperinflammatory, and no formal RCTs of follow-up immunotherapy have been performed to help to guide our decision; this is a substantial unmet need. However, such studies will be more challenging to perform than earlier trials, and until such data are available, one can rationally argue that the addition of alternative immunotherapies should be considered (Table 1 ).

It will thus be very important to pursue further clinical studies to identify novel immunotherapies that could improve the outcome of severe cases. If the patient is still not improving despite the available combinations, other immunomodulatory drugs could be an option to further dampen the hyperactive immune status, such as blocking C5a, anti-GM-CSF, or anti-TNF. However, the level of evidence for anti-cytokine therapies in the ICU patients beyond anti-IL- 6 is very low. Furthermore, an increased risk of secondary infections can be anticipated when blocking more components of the immune system. Therefore, escalation of immunosuppressive treatment is currently not advocated outside of clinical trials. An overview of novel potential therapies that need to be formally tested in future clinical trials is presented in Fig. 4.

A second quandary that has only been superficially addressed until now is represented by the pathophysiological heterogeneity of COVID-19. Several interventions proven to be effective work by modulating the host's immune response, or cascades downstream of the immune response. However, the host response to SARS-CoV-2 is complex, characterized by a plethora of pathways that can be both beneficial and deleterious. Not surprisingly, agents that modify these pathways can be beneficial for some patients and ineffective or even harmful in others. Further complexity arises when one considers that the agents themselves can have additive, multiplying, or negative effects when used in combination. These variable treatment effects, dependent on a patient's particular immune state, the disease course, and on the use of co-interventions, likely explain some of the disparate findings from some clinical trials. The weaving together of findings from these experiments into an overarching conceptual model is a largely theoretical exercise at this point. Consequently, the current evidence-based guidelines appear somewhat simplistic and lacking in nuance for the individualized treatments that many clinicians likely wish to prescribe. Nevertheless, 
Immunomodulatory treatment options according to WHO Clinical Progression Score

(1)

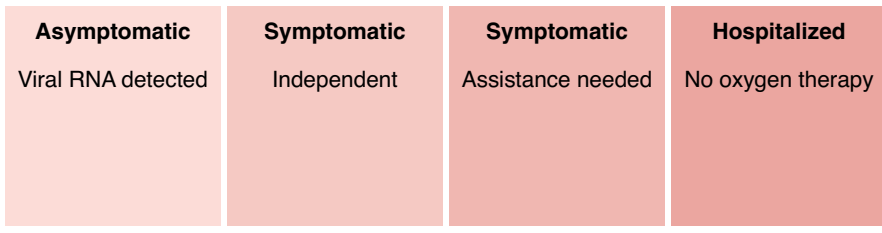

(5)

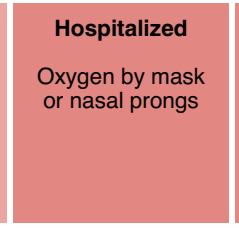

(6)

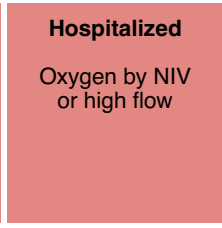

(7)

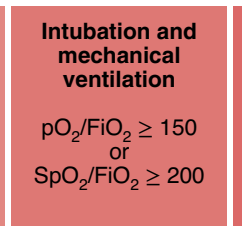

Dexamethasone

Anti-IL-1 (anakinra)

\section{Anti-IL-6 (tocilizumab, sarilumab)}

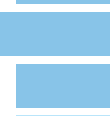

JAK inhibitors (baricitinib, tofacitinib)

Imatinib

Monoclonal antibodies (in seronegative patients)
Icatibant
Interferons/L-7/checkpoint inhibitors
Anti-TNF (adalimumab)/anti-GM-CSF
Anti-C5a (vilobelimab)

Fig. 4 | An overview of the options for immunotherapy in patients with COVID-19, depending on the stage of the disease (based on the WHO Clinical Progression Score). The treatments based on high-quality randomized trials are presented in dark blue, while the more speculative treatments based on observational or small case-series studies are presented in light blue. $\mathrm{FiO}_{2}$, fraction of inspired oxygen; GM-CSF, granulocyte-macrophage colony stimulating factor; IL-1/6, interleukin-1/6; JAK, Janus kinase; NIV, noninvasive ventilation; $\mathrm{pO}_{2}$, partial pressure of oxygen; SpO ${ }_{2}$, oxygen saturation; TNF, tumor necrosis factor.

preliminary data suggest that defined subgroups of patients (based on their inflammatory response) may benefit more, or less, from certain immunomodulatory therapies. The way forward is to perform trials based on robust biomarkers, so that patients that are more likely to benefit from a given treatment will receive it.

There are two broad barriers to the generation of robust experimental evidence supporting individualized treatment algorithms. First, the underlying heterogeneity in pathophysiology that likely drives differential treatment responses may often be clinically invisible: two clinically similar patients may have diverse immune states. Second, traditional trial designs are not well-suited for efficient evaluation of differential treatment effects in different patient groups. The good news is that much of the evidence supporting best treatment has come from adaptive platform trials, such as RECOVERY or REMAP-CAP. These designs are more flexible for the evaluation of combinations of therapies and evaluation of effects across different subgroups. And, indeed, one can argue that adaptive platform trials have been the dominant source of robust clinical evidence for COVID-19, perhaps ushering in a new paradigm for clinical research. Nonetheless, these trials have thus far still used relatively simple approaches for the assessment of subgroup effects and heterogeneity of treatment effects. Although they can provide clinical rationale and explore more personalized options when common approaches are not working, smaller trials often lack statistical power to confirm clinical efficacy. Therefore, immunotherapy in COVID-19 needs to be further explored through RCTs ito consolidate knowledge and experience and to determine the optimal biomarker-driven host-directed strategies.

One final quandary that must be addressed in the future is the availability of immunotherapy. Although the approaches described here can be incorporated in standard-of-care protocols of high-income countries, these treatments are often not available in many low- or middle-income countries. Efforts should be made to increase the availability of the current medications but also explore cheaper but equally effective alternatives. Only by ensuring equal therapeutic opportunities for all our patients can we fulfil our mission of optimal treatment of COVID-19.

Received: 25 August 2021; Accepted: 24 November 2021; Published online: 21 January 2022

\section{References}

1. Walls, A. C. et al. Structure, function, and antigenicity of the SARS-CoV-2 spike glycoprotein. Cell 181, 281-292.e6 (2020).

2. Hoffmann, M. et al. SARS-CoV-2 Cell entry depends on ACE2 and TMPRSS2 and is blocked by a clinically proven protease inhibitor. Cell 181, 271-280 (2020).

3. Hou, Y. J. et al. SARS-CoV-2 reverse genetics reveals a variable infection gradient in the respiratory tract. Cell 182, 429-446.e14 (2020).

4. Jouan, Y., Baranek, T., Si-Tahar, M., Paget, C. \& Guillon, A. Lung compartmentalization of inflammatory biomarkers in COVID-19-related ARDS. Crit. Care 25, 120 (2021).

5. Giamarellos-Bourboulis, E. J. et al. Complex immune dysregulation in COVID-19 patients with severe respiratory failure. Cell Host Microbe 27, 992-1000.e3 (2020).

6. Chen, $\mathrm{X}$. et al. Detectable serum severe acute respiratory syndrome coronavirus 2 viral load (RNAemia) is closely correlated with drastically elevated interleukin 6 level in critically ill patients with coronavirus disease 2019. Clin. Infect. Dis. 71, 1937-1942 (2020).

7. Chen, G. et al. Clinical and immunological features of severe and moderate coronavirus disease 2019. J. Clin. Invest. 130, 2620-2629 (2020).

8. Payen, D. et al. A longitudinal study of immune cells in severe COVID-19 patients. Front. Immunol. 11, 580250 (2020).

9. Huang, I. \& Pranata, R. Lymphopenia in severe coronavirus disease-2019 (COVID-19): systematic review and meta-analysis. J. Intensive Care 8, 1-10 (2020).

10. Iskander, K. N. et al. Sepsis: multiple abnormalities, heteroge-neous responses, and evolving understanding. Physiol. Rev. 93, 1247-1288 (2013).

11. Du, R. H. et al. Predictors of mortality for patients with COVID-19 pneumonia caused by SARSCoV- 2: a prospective cohort study. Eur. Respir. J. 55, 2000524 (2020).

12. Janssen, N. A. F. et al. Dysregulated innate and adaptive immune responses discriminate disease severity in COVID-19. J. Infect. Dis. 223, 1322-1333 (2021).

13. Laing, A. G. et al. A dynamic COVID-19 immune signature includes associations with poor prognosis. Nat. Med. 26, 1623-1635 (2020). 
14. Kreutmair, S. et al. Distinct immunological signatures discriminate severe COVID-19 from non-SARS-CoV-2-driven critical pneumonia. Immunity 54, 1578-1593.e5 (2021).

15. Bost, P. et al. Deciphering the state of immune silence in fatal COVID-19 patients. Nat. Commun. 12, 1-15 (2021).

16. Obi, A. T., Barnes, G. D., Napolitano, L. M., Henke, P. K. \& Wakefield, T. W. Venous thrombosis epidemiology, pathophysiology, and anticoagulant therapies and trials in severe acute respiratory syndrome coronavirus 2 infection. J. Vasc. Surg. Venous Lymphat. Disord. 9, 23-35 (2021).

17. Zhou, F. et al. Clinical course and risk factors for mortality of adult inpatients with COVID-19 in Wuhan, China: a retrospective cohort study Lancet 395, 1054-1062 (2020).

18. Chen, T. et al. Clinical characteristics of 113 deceased patients with coronavirus disease 2019: Retrospective study. Brit. Med. J. 368, m1295 (2020).

19. Goyal, P. et al. Clinical Characteristics of Covid-19 in New York City. N. Engl. J. Med. 382, 2372-2374 (2020).

20. Mandal, S. et al. 'Long-COVID': a cross-sectional study of persisting symptoms, biomarker and imaging abnormalities following hospitalisation for COVID-19. Thorax 76, 396-398 (2021).

21. Bloch, E. M. et al. Deployment of convalescent plasma for the prevention and treatment of COVID-19. J. Clin. Invest. 130, 2757-2765 (2020).

22. Shankar-Hari, M., Spencer, J., Sewell, W. A., Rowan, K. M. \& Singer, M. Bench-to-bedside review: Immunoglobulin therapy for sepsis - biological plausibility from a critical care perspective. Crit. Care 16, 206 (2012).

23. Liu, L. et al. Anti-spike IgG causes severe acute lung injury by skewing macrophage responses during acute SARS-CoV infection. JCI Insight 4, e123158 (2019).

24. Bournazos, S., Gupta, A. \& Ravetch, J. V. The role of IgG Fc receptors in antibody-dependent enhancement. Nat. Rev. Immunol. 20, 633-643 (2020).

25. Zinsser H., Batchelder A. P. Studies on Mexican typhus fever. I. J. Exp. Med. 51, 847-858 (1930).

26. O'Donnell, M. R. et al. A randomized double-blind controlled trial of convalescent plasma in adults with severe COVID-19. J. Clin. Invest. 131, e150646 (2021).

27. Arnold Egloff, S. A. et al. Convalescent plasma associates with reduced mortality and improved clinical trajectory in patients hospitalized with COVID-19. J. Clin. Invest. 131, e151788 (2021).

28. Senefeld, J. W. et al. Use of convalescent plasma in COVID-19 patients with immunosuppression. Transfusion 61, 2503-2511 (2021).

29. Piechotta, V. et al. Convalescent plasma or hyperimmune immunoglobulin for people with COVID-19: a living systematic review. Cochrane Database Syst. Rev. 7, 1-293 (2020).

30. Abani, O. et al. Convalescent plasma in patients admitted to hospital with COVID-19 (RECOVERY): a randomised controlled, open-label, platform trial. Lancet 397, 2049-2059 (2021).

31. Writing Committee for the REMAP-CAP Investigators et al. Effect of convalescent plasma on organ support-free days in critically ill patients with COVID-19: a randomized clinical trial. JAMA 326, 1690-1702 (2021).

32. Aiello, A. et al. Immunosenescence and Its hallmarks: how to oppose aging strategically? A review of potential options for therapeutic intervention. Front. Immunol. 10, 2247 (2019).

33. Libster, R. et al. Early high-titer plasma therapy to prevent severe COVID-19 in older adults. N. Engl. J. Med. 384, 610-618 (2021).

34. Gottlieb, R. L. et al. Effect of bamlanivimab as monotherapy or in combination with etesevimab on viral load in patients with mild to moderate COVID-19. JAMA 325, 632-644 (2021).

35. RECOVERY Collaborative Group. Casirivimab and imdevimab in patients admitted to 4 hospital with COVID-19 (RECOVERY): a randomised, controlled, open-label, platform trial. Preprint at medRxiv https://doi. org/10.1101/2021.06.15.21258542 (2021).

36. Mathew, D. et al. Deep immune profiling of COVID-19 patients reveals distinct immunotypes with therapeutic implications. Science 369, eabc8511 (2020).

37. Wu, C. et al. Risk factors associated with acute respiratory distress syndrome and death in patients with coronavirus disease 2019 pneumonia in wuhan, China. JAMA Intern. Med. 180, 934-943 (2020).

38. Horby, P. et al. Dexamethasone in hospitalized patients with COVID-19. N. Engl. J. Med. 384, 693-704 (2021).

39. Angus, D. C. et al. Effect of hydrocortisone on mortality and organ support in patients with severe COVID-19. JAMA 324, 1330-1341 (2020).

40. Tomazini, B. M. et al. Effect of dexamethasone on days alive and ventilator-free in patients with moderate or severe acute respiratory distress syndrome and COVID-19. JAMA 324, 1307-1316 (2020).

41. Sterne, J. A. C. et al. Association between administration of systemic corticosteroids and mortality among critically ill patients with COVID-19. JAMA 324, 1330-1341 (2020).

42. Rochwerg, B. et al. A living WHO guideline on drugs for COVID-19. Brit. Med. J. 370, m3379 (2020).
43. Ferguson, F. M. \& Gray, N. S. Kinase inhibitors: the road ahead. Nat. Rev. Drug Discov. 17, 353-377 (2018).

44. Jacobs, C. F., Eldering, E. \& Kater, A. P. Kinase inhibitors developed for treatment of hematologic malignancies: implications for immune modulation in COVID-19. Blood Adv. 5, 913-925 (2021).

45. Kalil, A. C. et al. Baricitinib plus remdesivir for hospitalized adults with COVID-19. N. Engl. J. Med. 384, 795-807 (2021).

46. Marconi, V. C. et al. Efficacy and safety of baricitinib for the treatment of hospitalised adults with COVID-19 (COV-BARRIER): a randomised, double-blind, parallel-group, placebo-controlled phase 3 trial. Lancet Respir Med. 19, 1-12 (2021).

47. Aman, J. et al. Imatinib in patients with severe COVID-19: a randomised, double-blind, placebo-controlled, clinical trial. Lancet Respir. Med. 9, 957-968 (2021).

48. Guimarães, P. O. et al. Tofacitinib in patients hospitalized with COVID-19 pneumonia. N. Engl. J. Med. 385, 406-415 (2021).

49. Tharaux, P.-L. et al. Effect of anakinra versus usual care in adults in hospital with COVID-19 and mild-to-moderate pneumonia (CORIMUNO-ANA-1): a randomised controlled trial. Lancet Respir. Med. 9, 295-304 (2021).

50. The REMAP-CAP Investigators. Effectiveness of tocilizumab, sarilumab, and anakinra for critically ill patients with COVID-19 The REMAP-CAP COVID-19 Immune Modulation Therapy Domain Randomized Clinical Trial. Preprint at medRxiv https://doi.org/10.1101/2021.06.18.21259133 (2021).

51. Rovina, N. et al. Soluble urokinase plasminogen activator receptor (suPAR) as an early predictor of severe respiratory failure in patients with COVID-19 pneumonia. Crit. Care 24, 187 (2020).

52. Azam, T. U. et al. Soluble urokinase receptor (SuPAR) in COVID-19-related AKI. J. Am. Soc. Nephrol. 31, 2725-2735 (2020).

53. Kyriazopoulou, E. et al. An open label trial of anakinra to prevent respiratory failure in COVID-19. eLife 10, e66125 (2021).

54. Kyriazopoulou, E. et al. Early treatment of COVID-19 with anakinra guided by soluble urokinase plasminogen receptor plasma levels: a double-blind, randomized controlled phase 3 trial. Nat. Med. 27, 1752-1760 (2021).

55. Caricchio, R. et al. Effect of canakinumab vs placebo on survival without invasive mechanical ventilation in patients hospitalized with severe COVID-19. JAMA 326, 230-239 (2021).

56. Abani, O. et al. Tocilizumab in patients admitted to hospital with COVID-19 (RECOVERY): a randomised, controlled, open-label, platform trial. Lancet 397, 1637-1645 (2021).

57. Gordon, A. C. et al. Interleukin-6 receptor antagonists in critically ill patients with COVID-19. N. Engl. J. Med. 384, 1491-1502 (2021).

58. Domingo, P. et al. Association between administration of IL-6 antagonists and mortality among patients hospitalized for COVID-19. JAMA 326, 499-518 (2021).

59. Patel, J. et al. A randomized trial of otilimab in severe COVID-19 pneumonia (OSCAR). Preprint at medRxiv https://doi org/10.1101/2021.04.14.21255475 (2021).

60. Diao, B. et al. Human kidney is a target for novel severe acute respiratory syndrome coronavirus 2 infection. Nat. Commun. 12, 2506 (2021).

61. Schurink, B. et al. Viral presence and immunopathology in patients with lethal COVID-19: a prospective autopsy cohort study. Lancet Microbe 1, e290-e299 (2020).

62. Seshan, S. V. et al. Role of tissue factor in a mouse model of thrombotic microangiopathy induced by antiphospholipid antibodies. Blood 114, 1675-1683 (2009).

63. Kambas, K. et al. C5a and TNF- $\alpha$ up-regulate the expression of tissue factor in intra-alveolar neutrophils of patients with the acute respiratory distress syndrome. J. Immunol. 180, 7368-7375 (2008).

64. Ritis, K. et al. A novel C5a receptor-tissue factor cross-talk in neutrophils links innate immunity to coagulation pathways. J. Immunol. 177, 4794-4802 (2006).

65. de Bruin, S. et al. Clinical features and prognostic factors in COVID-19: a prospective cohort study. EBioMedicine 67, 103378 (2021).

66. Vlaar, A. P. J. et al. Anti-C5a antibody IFX-1 (vilobelimab) treatment versus best supportive care for patients with severe COVID-19 (PANAMO): an exploratory, open-label, phase 2 randomised controlled trial. Lancet Rheumatol. https://doi.org/10.1016/S2665-9913(20)30341-6 (2020).

67. Cinatl, J. et al. Treatment of SARS with human interferons. Lancet 362 , 293-294 (2003).

68. Monk, P. D. et al. Safety and efficacy of inhaled nebulised interferon beta-1a (SNG001) for treatment of SARS-CoV-2 infection: a randomised, double-blind, placebo-controlled, phase 2 trial. Lancet Respir. Med. 9, e764-e773 (2021).

69. Hung, I. F. N. et al. Triple combination of interferon beta-1b, lopinavirritonavir, and ribavirin in the treatment of patients admitted to hospital with COVID-19: an open-label, randomised, phase 2 trial. Lancet 395, 1695-1704 (2020). 
70. Pan, H. et al. Repurposed antiviral drugs for COVID-19 - interim WHO Solidarity Trial results. N. Engl. J. Med. 384, 497-511 (2021).

71. van Laarhoven, A. et al. Interferon gamma immunotherapy in five critically ill COVID-19 patients with impaired cellular immunity: a case series. Med (N Y.) 2, 1163-1170 (2021).

72. Nguyen, L. S., Ait Hamou, Z., Gastli, N., Chapuis, N. \& Pène, F. Potential role for interferon gamma in the treatment of recurrent ventilator-acquired pneumonia in patients with COVID-19: a hypothesis. Intensive Care Med. 47, 619-621 (2021)

73. Laterre, P. F. et al. Association of interleukin 7 immunotherapy with lymphocyte counts among patients with severe coronavirus disease 2019 (COVID-19). JAMA Netw. Open 3, 3-7 (2020).

74. Shatzel, J. J. et al. The contact activation system as a potential therapeutic target in patients with COVID-19. Res. Pract. Thromb. Haemost. 4, 500-505 (2020).

75. van de Veerdonk, F. L. et al. Kallikrein-kinin blockade in patients with COVID-19 to prevent acute respiratory distress syndrome. eLife $\mathbf{9}, 57555$ (2020).

76. van de Veerdonk, F. L. et al. Outcomes associated with use of a kinin B2 receptor antagonist among patients with COVID-19. JAMA Netw. Open 3 e2017708 (2020)

77. Mansour, E. et al. Evaluation of the efficacy and safety of icatibant and C1 esterase/kallikrein inhibitor in severe COVID-19: study protocol for a three-armed randomized controlled trial. Trials 22, 1-13 (2021).

78. Bonaventura, A. et al. Endothelial dysfunction and immunothrombosis as key pathogenic mechanisms in COVID-19. Nat. Rev. Immunol. 21, 319-329 (2021)

79. Talasaz, A. H. et al. Recent randomized trials of antithrombotic therapy for patients with COVID-19. J. Am. Coll. Cardiol. 77, 1903-1921 (2021).

80. Muñoz-Rivas, N. et al. Systemic thrombosis in a large cohort of COVID-19 patients despite thromboprophylaxis: a retrospective study. Thromb. Res. 199, 132-142 (2021).

81. Mazloomzadeh, S. et al. Effect of intermediate-dose vs standard-dose prophylactic anticoagulation on thrombotic events, extracorporeal membrane oxygenation treatment, or mortality among patients with COVID-19 admitted to the intensive care unit. JAMA 325, 1620-1630 (2021).

82. Lopes, R. D. et al. Therapeutic versus prophylactic anticoagulation for patients admitted to hospital with COVID-19 and elevated D-dimer concentration (ACTION): an open-label, multicentre, randomised, controlled trial. Lancet 397, 2253-2263 (2021).

83. ATTACC Investigators et al. Therapeutic anticoagulation with heparin in noncritically ill patients with COVID-19. N. Engl. J. Med. 385, 790-802 (2021).

84. REMAP-CAP Investigators et al. Therapeutic anticoagulation with heparin in critically ill patients with COVID-19. N. Engl. J. Med. 385, 777-789 (2021).

85. Bendib, I. et al. Alveolar compartmentalization of inflammatory and immune cell biomarkers in pneumonia-related ARDS. Crit. Care 25, 23 (2021).

86. Richeldi, L. et al. Efficacy of a tyrosine kinase inhibitor in idiopathic pulmonary fibrosis. N. Engl. J. Med. 365, 1079-1087 (2011).

87. Richeldi, L. et al. Efficacy and safety of nintedanib in patients with advanced idiopathic pulmonary fibrosis. BMC Pulm. Med. 20, 3 (2020).

88. Menshawey, R., Menshawey, E., Alserr, A. H. K. \& Abdelmassih, A. F. JAK out of the box; the rationale behind Janus kinase inhibitors in the COVID-19 setting, and their potential in obese and diabetic populations. Cardiovasc. Endocrinol. Metab. 10, 80-88 (2021).

89. Henderson, L. A. et al. American College of Rheumatology clinical guidance for multisystem inflammatory syndrome in children associated with SARS-CoV-2 and hyperinflammation in pediatric COVID-19: version 1. Arthritis Rheumatol. 72, 1791-1805 (2020).

90. Pouletty, M. et al. Paediatric multisystem inflammatory syndrome temporally associated with SARS-CoV-2 mimicking Kawasaki disease (KawaCOVID-19): a multicentre cohort. Ann. Rheum. Dis. 79, 999-1006 (2020).

91. Feldstein, L. R. et al. Multisystem inflammatory syndrome in U.S. children and adolescents. N. Engl. J. Med. 383, 334-346 (2020).

92. Toubiana, J. et al. Kawasaki-like multisystem inflammatory syndrome in children during the COVID-19 pandemic in Paris, France: prospective observational study. Brit. Med. J. 369, m2094 (2020).
93. Cheung, E. W. et al. Multisystem inflammatory syndrome related to COVID-19 in previously healthy children and adolescents in New York City. JAMA 324, 294 (2020).

94. Ramcharan, T. et al. Paediatric inflammatory multisystem syndrome: temporally associated with SARS-CoV-2 (PIMS-TS): cardiac features, management and short-term outcomes at a UK Tertiary Paediatric Hospital Pediatr. Cardiol. 41, 1391-1401 (2020).

95. Nakra, N., Blumberg, D., Herrera-Guerra, A. \& Lakshminrusimha, S. Multi-system inflammatory syndrome in children (MIS-C) following SARS-CoV-2 infection: review of clinical presentation, hypothetical pathogenesis, and proposed management. Children 7, 69 (2020).

96. Lee, P. Y. et al. Distinct clinical and immunological features of SARS-CoV-2-induced multisystem inflammatory syndrome in children. $J$. Clin. Invest. 130, 5942-5950 (2020).

97. Whittaker, E. et al. Clinical characteristics of 58 children with a pediatric inflammatory multisystem syndrome temporally associated with SARS-CoV-2. JAMA 324, 259-269 (2020).

98. Riollano-Cruz, M. et al. Multisystem inflammatory syndrome in children related to COVID-19: A New York City experience. J. Med. Virol. 93, 424-433 (2021).

99. Ruscitti, P. et al. Severe COVID-19, another piece in the puzzle of the hyperferritinemic syndrome. An immunomodulatory perspective to alleviate the storm. Front. Immunol. 11, 1130 (2020).

100. Morris, S. B. et al. Case series of multisystem inflammatory syndrome in adults associated with SARS-CoV-2 infection - United Kingdom and United States, March-August 2020. MMWR. Morb. Mortal. Wkly. Rep. 69 , 1450-1456 (2020)

101. Bastug, A. et al. Multiple system inflammatory syndrome associated with SARS-CoV-2 infection in an adult and an adolescent. Rheumatol. Int. 41, 993-1008 (2021).

102. Uwaydah, A. K., Hassan, N. M. M., Abu Ghoush, M. S. \& Shahin, K. M. M. Adult multisystem inflammatory syndrome in a patient who recovered from COVID-19 postvaccination. BMJ Case Rep. 14, e242060 (2021).

103. Son, M. B. F. et al. Multisystem inflammatory syndrome in children - initial therapy and outcomes. N. Engl. J. Med. 385, 11-22 (2021).

104. McArdle, A. J. et al. Treatment of multisystem inflammatory syndrome in children. N. Engl. J. Med. 385, 11-22 (2021).

105. Ouldali, N. et al. Association of intravenous immunoglobulins plus methylprednisolone vs immunoglobulins alone with course of fever in multisystem inflammatory syndrome in children. JAMA 325, 855-864 (2021).

106. Döcke, W. D. et al. Monocyte deactivation in septic patients: restoration by IFN- $\gamma$ treatment. Nat. Med. 3, 678-681 (1997).

\section{Acknowledgements}

F.L.v.d.V. gratefully acknowledges financial support from the Netherlands Organization for Scientific Research (Vidi grant from ZonMW \& ZonMW project nr 10430012010010).M.G.N. was supported by a Spinoza grant of the Netherlands Association for Scientific Research.

\section{Competing interests}

The authors declare no competing interests.

\section{Additional information}

Correspondence should be addressed to Frank L. van de Veerdonk or Mihai G. Netea. Peer review information Nature Medicine thanks Michael Joyner and Vincenzo Bronte for their contribution to the peer review of this work. Karen O'Leary was the primary editor on this article and managed its editorial process and peer review in collaboration with the rest of the editorial team.

Reprints and permissions information is available at www.nature.com/reprints. Publisher's note Springer Nature remains neutral with regard to jurisdictional claims in published maps and institutional affiliations.

(c) Springer Nature America, Inc. 2022 